\title{
ON A COMMON ABSTRACTION OF BOOLEAN RINGS AND LATTICE ORDERED GROUPS II
}

\author{
V. V. RAMA RAO \\ (Received 6 August 1969; revised 10 December 1969) \\ Communicated by B. Mond
}

\section{Introduction}

In an earlier paper, the author has obtained a solution [8] to Birkhoff's problem No. 105 [1]: Is there a common abstraction which includes Boolean algebras (Rings) and lattice ordered groups as special cases? The solution actually turns out to be the direct product of a Boolean ring and a lattice ordered group. Birkhoff's problem has also been solved by Swamy, Wyler, and Nakano by presenting respectively i) Dually Residuated Lattice Ordered Semigroups (D.R.1. semigroups) $[2,3,4,5]$, ii) Clans [6], and iii) Multirings [7].

In this paper, starting with a lattice $L$ and a single binary operation '- ' (just as in clans) satisfying (1.1) through (1.5) (see definition below), we obtain one more common abstraction which we call a Boolean-l-algebra (Booleanlattice-algebra); and which has more geometric structure than D.R.1. semigroups. Also a Boolean-l-algebra turns out to be a clan with a total operation ' -'. Thus a Boolean- $l$-algebra is an improvement on clans, since by definition a clan is a lattice with a partial binary operation. It may also be mentioned here that conditions 3 and 4 of the Main Theorem in [8] are too cumbersome.

It turns out that the class of Boolean- $l$-algebras is a subclass of D.R.1. semigroups also. Thus all the results of D.R.l. semigroups are valid here also, and in addition a Boolean-l-algebra has interesting geometric properties (see section 3 ) which in general are not true in D.R.l. semigroups. The reason for this situation is not difficult to find. For D.R.l. semigroups include Brouwerian algebras also, and many geometric properties common to Boolean rings and commutativelattice ordered groups will not hold in Brouwerian algebras. For instance, lattice betweenness and metric betweenness are equivalent in both Boolean rings and commutative-l-groups, but not in Brouwerian algebras. Thus it looks as though the presence of Brouwerian algebras in D.R.l. semigroups is responsible for the smaller number of geometric results for D.R.l. semigroups. We establish that this is indeed the case by showing that Brouwerian algebras are completely excluded from being Boolean- $l$-algebras. 
If $A=(A ; \cup, \cap,+,-)$ is a D.R.l. semigroup, then $(A ; \cup, \cap,-)$ fails to be a clan even if ' - ' is restricted suitably. Since a Boolean- $l$-algebra is also a clan, the class of Boolean- $l$-algebras may also be looked upon as the subclass of D.R.I. semigroups which are also clans simultaneously.

This paper is divided into three sections. In section 1, we define a Booleanl-algebra, give examples, and show that the axioms are independent. Here we investigate the possibility of the class of Brouwerian algebras being a subclass of Boolean-l-algebras, and show that this is never the case. In section 2, we show that a Boolean-l-algebra is both a D.R.l. semigroup and a clan, while section 3 is devoted to establishing the geometric properties.

Lastly, this paper is completely independent of the author's earlier work in [8].

\section{1.}

Definition 1. A lattice $L=(L ; \cup, \cap)$ with a binary operation ' - ' is called a Boolean-l-algebra iff

(1.1.) $a \cup b-c=(a-c) \cup(b-c)$,

(1.2.) $a-(b \cap c)=(a-b) \cup(a-c)$, $a-(b \cup c)=(a-b) \cap(a-c)$,

(1.3.) If $a \leqq b$, then $c-b=(c-a)-(b-a)$,

(1.4.) If $a \geqq b \cup c$, then $a-b \geqq a-c$ implies $c \geqq b$,

(1.5.) Given $a$ and $b$, there exists $c$ in $L$ such that $p-c=(p-b)-a$ for all $p$. Note that (1.5) is not needed in this section.

We shall now give examples of Boolean- $l$-algebras.

1) If $B=(B ;+, \cap)$ is a Boolean ring then $(B ;-)$ is a Boolean-l-algebra if we define $a-b=a+a \cap b$. Verification is routine.

2) If $A=(A ; \cup, \cap,+)$ is a lattice ordered group, then $(A ; \cup, \cap,-)$ is a Boolean- $l$-algebra, where ' - ' is the usual group difference.

3 ) If $N$ is the set of positive integers with ' 0 ', then $(N ;-)$ with the usual ordering is a Boolean- $l$-algebra with ' -' defined as follows: If $a \geqq b$ then $a-b=$ usual difference, otherwise $a-b=0$.

4) Any D.R.l. semigroup $A$ is a Boolean- $l$-algebra if

i) $a-(b \cup c)=(a-b) \cap(a-c)$,

ii) If $a \geqq b \cup c$ and $a-b \geqq a-c$ then $c \geqq b$.

The following examples show that (1.3) and (1.4) are essentially independent of the rest. 
[1.3]. Let $L$ be any $l$-group. Define $a-b=-b$ for $a, b \in L$. Then (1.1), (1.2), and (1.4) are satisfied and (1.3) fails.

[1.4] Let $L$ be any chain with ' 0 ' as least element. Define $a-b=a$ if $a \geqq b$ and $a-b=0$ otherwise. Then (1.1), (1.2), and (1.3) are satisfied but (1.4) fails.

We shall now study the consequences of our definition. Throughout this paper $L$ stands for a Boolean- $l$-algebra and $a, b, c, x, y, t \cdots$ denote the elements of $L$.

Lemma 1. If $b \cap c \geqq a$ and $b-a \leqq c-a$ then $b \leqq c$.

Proof. Put $t=b \cup c$. Then by (1.3) and (1.2)

$$
t-c=(t-a)-(c-a) \leqq(t-a)-(b-a)=t-b
$$

so that $b \leqq c$ by $(1.4)$.

LemMA 2. The equation $x-x=x$ has a unique solution, denoted by 0 .

Proof. Obviously by (1.3), $a-a$ is a solution of this equation, for any $a$. If $x$ and $y$ are any two solutions, put $t=x \cup y$. Then $t \cap(t-x) \geqq x$ by (1.1) and $t-x=(t-x)-x$ by (1.3), so that $t-x=t$ by lemma 1. Similarly $t-y=t$ and by (1.4) $x=y$.

COROLlary 1. If $t \geqq 0$, then $t-0=t$.

Corollary 2. $a-a \cap b=a \cup b-b$.

LEMMA 3. Lis a distributive lattice.

PRoof. If $a \cup b=a \cup c$ and $a \cap b=a \cap c$, then by corollary above, and (1.4) it follows that $b=c$. Hence $\mathrm{L}$ is a distributive lattice.

LEMMA 4. If $a \geqq b$ and $a-b=0$, then $a=b$.

Proof. $a-b \leqq b-b$ and $a \cap b=b$, and so by lemma $1, a \leqq b$.

The following theorem gives the degeneracy of $L$ into a Boolean ring. However, its main use is in the subsequent corollary.

THEOREM 1. L is a Boolean ring iff $x \leqq a$ implies $x \cap(a-x)=0$.

Proof. The first part is clear. For the other part we need several lemmas, and from now on we assume $x \leqq a$ implies $x \cap(a-x)=0$.

LEMMA 5. ' 0 ' is the least element of $L$ and $a-0=a$ for all $a \in L$.

Proof. Since $a \leqq a$ for all $a, 0=a \cap(a-a)=a \cap 0$. Therefore $a-0=a$ for all $a$, by corollary 1 .

LEMMA 6. If $x \leqq a$, then $a-x=(a-x)-x$.

Proof. $a-x=(a-x)-\{(a-x) \cap x\}=0 \cup\{(a-x)-x\}=(a-x)-x$. 
LeMmA 7. $x \cup(a-x)=a$ if $x \leqq a$.

Proof. $\{x \cup(a-x)\}-x=a-x$, by (1.1) and lemmas 5 and 6 .

Hence $x \cup(a-x)=a$, by lemma 1 .

COROLlaRY 3. L is a section complemented lattice.

LEMMA 8. L is a Boolean ring.

Proof. $L$ is distributive (lemma 3 ) and section complemented by the above corollary. Let $x<z<y$. Then

$$
\begin{aligned}
z \cap\{x \cup(y-z)\} & =(z \cap x) \cup\{z \cap(y-z)\} \\
& =(z \cap x) \cup 0(\text { by hypothesis })=x
\end{aligned}
$$

and

$$
z \cup\{x \cup(y-z)\}=x \cup z \cup(y-z)=x \cup y(\text { by lemma 7) }=y .
$$

Hence $L$ is relatively complemented and so $L$ is a Boolean ring.

Lemma 8 completes the proof of theorem 1 .

COROLlary 4. If $(B ;-)$ is a Brouwerian algebra, then $(B ;-)$ is a Booleanl-algebra iff $B$ is a Boolean ring.

Proof. Let $(B ;-)$ be a Brouwerian algebra, satisfying (1.1) through (1.4). If $x \leqq a$, then $a=a \cup x=x \cup(a-x)$ since $(B ;-)$ is a Brouwerian algebra. Further,

$$
a-x=\{x \cup(a-x)\}-x=(a-x)-\{x \cap(a-x)\} \text { (by corollary 2) }
$$

and hence $x \cap(a-x)=0$ by 1.4. Therefore by theorem $1, B$ is a Boolean ring. The converse is routine.

2.

This section is devoted to establishing that a Boolean-l-algebra is both a D.R.l. semigroup and a clan. We first show that (1.5) is independent of (1.1) through (1.4).

[1.5]. Let $A$ be the set of all integers $\geqq-p$ where $p>0$. Define $a-b=$ usual difference if $a \geqq b$, otherwise $a-b=0$. Then (1.1) through (1.4) hold in $A$ with the usual ordering; but (1.5) fails.

From now onwards we assume that $L$ satisfies (1.5) also.

Remark 1. The ' $c$ ' which satisfies the equation (1.5) is unique by (1.4) and we denote this by $a+b$.

REMARK 2. If ' + ' is commutative then $a+b=a \cup b+a \cap b$. 
In order to show that a Boolean-l-algebra is a D.R.l. semigroup, we need some preparation.

LEMMA 9. $(a-b) \cup 0+b=a \cup b$ and hence $0+b=b$.

Proof. By (1.3)

$$
c-(a \cup b)=(c-b)-(a \cup b-b)=c-\{(a \cup b-b)+b\} .
$$

Hence $a \cup b=(a \cup b-b)+b=(a-b) \cup 0+b$.

Corollary 5. $a-b \leqq 0$ iff $a \leqq b$.

Proof. By (1.2) and lemma 9.

COROLlaRy 6. $(b-a)+a \geqq b$

Corollary 7. $(x+y)-y \leqq x$.

Proof. Put $a=(x+y)-y$ and $b=x$ in corollary 5 .

LeMma 10. $(a+b)+c=a+(b+c)$.

Proof. We have for all $p$,

$$
\begin{aligned}
p-\{a+(b+c)\} & =\{p-(b+c)\}-a=\{(p-c)-b\}-a \\
& =(p-c)-(a+b)=p-\{(a+b)+c\} .
\end{aligned}
$$

Hence the lemma.

LEMMA 11. $a \cup b+c=(a+c) \cup(b+c)$ and $a \cap b+c=(a+c) \cap(b+c)$.

PROOF.

$$
\begin{aligned}
p-\{(a+c) \cup(b+c)\} & =\{p-(b+c)\} \cap\{p-(a+c)\} \\
& =\{(p-c)-b\} \cap\{(p-c)-a\}=(p-c)-(a \cup b) \\
& =p-\{(a \cup b)+c\} .
\end{aligned}
$$

Hence $a \cup b+c=(a+c) \cup(b+c)$. Similarly $a \cap b+c=(a+c) \cap(b+c)$.

LEMMA 12. $a \cap b-c=(a-c) \cap(b-c)$.

PROOF. $(a-c) \cap(b-c)-\{(a \cap b)-c\}$

$$
\begin{aligned}
& \leqq[(a-c)-\{(a \cap b)-c\}] \cap[(b-c)-\{(a \cap b)-c\}] \\
& =[a-\{(a \cap b-c)+c\}\} \cap[b-\{(a \cap b-c)+c\}] \\
& \leqq(a-a \cap b) \cap(b-a \cap b) \text { (by corollary 6) }=0 .
\end{aligned}
$$

Therefore $(a-c) \cap(b-c) \leqq a \cap b-c$. The other part is by (1.1).

LEMMA 13. $c+a \cup b=(c+a) \cup(c+b)$ and $c+a \cap b=(c+a) \cap(c+b)$.

Proof. On the same lines of lemma 11, using lemma 12 and (1.1). 
LEMMA 14. $a-0=a=a+0$ for all $a$.

PRoOF. Let $p=(a+0) \cup a \cup 0$. Then

$$
p-(a+0)=(p-0)-a=p-a
$$

by lemma 2 , and hence $a+0=a$ by (1.4). Also

by corollary 7 and

$$
a-0=(a+0)-0 \leqq a
$$

by corollary 6 .

$$
a-0=(a-0)+0 \geqq a
$$

Lemmas $10,11,13$ and 14 will establish $(L ; \cup, \cap,+)$ as a lattice ordered semigroup with ' 0 ' and by lemmas 2,9 and corollaries 6 and 7 we obtain the following by theorem 1.1 of [5].

Theorem 2. If $(L ; \cup, \cap,-)$ is a Boolean-l-algebra, then $(L ; \cup, \cap,-)$ is a Dually Residuated lattice ordered semigroup.

We shall now show that $L$ is a clan. In fact

Lemma 15. If $A=(A ; \cup, \cap,+,-)$ is a Dually Residuated lattice ordered semigroup, with $a-(b \cup c)=(a-b) \cap(a-c)$, then $(A ; \cup, \cap,-)$ is a clan iff $(A ; \cup, \cap,-)$ is a Boolean-l-algebra.

Proor. Let $\sum=\{(a, b) \in L \times L \mid a \leqq b\}$, and $\sigma: \sum \rightarrow L$ be defined by $\sigma(a, b)=b-a$. It is a routine verification that $C_{1}, C_{2}, C_{3}, C_{4}$ and $C_{7}$ (see [6]) are satisfied.

Conversely let $\sum=\{(a, b) \in A \times A \mid a \leqq b\}$ and $\sigma: \sum \rightarrow A$ be defined by $\sigma(a, b)=b-a$ and let $C_{1}, C_{2}, C_{3}, C_{4}$ and $C_{7}$ be satisfied in $A$. We have only to show that (1.4) holds in $A$ on account of lemmas 6, 7, 8 and 11 of [5]. But (1.4) is precisely $C_{2}$ and so $A$ is a Boolean- $l$-algebra.

\section{3.}

In this section we study the geometry of a commutative Boolean- $l$-algebra; i.e. we assume ' + ' is commutative.

${ }^{1}$ A system $A=(A ;+$, $\leqq-)$ is called a Dually Residuated lattice ordered semigroup iff

(1.1) $(A ;+, \leqq)$ is a lattice ordered semigroup with " 0 " i.e. $a+(b \cup c)=(a+b) \cup(a+c)$ and $a+(b \cap c)=(a+b) \cap(a+c)$ and $a \cup b+c=(a+c) \cup(b+c)$ and $a \cap b+c=$ $(a+c) \cap(b+c)$ for all $a, b, c \in A$. $a-b$.

(1.2). Given $a, b$ in $A$ there exists a least $x$ in $A$ such that $x+b \geqq a$ we denote this $x$ by

(1.3). $(a-b) \cup 0+b \leqq a \cup b$ and,

(1.4). $a-a \geqq 0$.

Th 1.1 [5]: Any Dually Residuated lattice ordered semigroup $A=(A ;+, \leqq-)$ can be equationally defined as an algebra with the binary operations $+, \cup, \cap,-$ replacing (1.2) by the equations (1.2.1) $(y-x)+x \geqq y(1.2 .2) x-y \leqq x \cup z-y$ and (1.2.3) $(x+y)-y \leqq x$. 
Since $L$ is a D.R.I. semigroup by theorem 2 , it is also an autometrized lattice $[2,3]$ with distance function $d: L \times L \rightarrow L$ defined by $d(a, b)=a * b=(a-b)$ $\cup(b-a)$. Also it is an autometrized lattice with distance $d(a, b)=(a-b) \cup 0+$ $(b-a) \cup 0$. The following lemma shows that these two metrics will coincide in $L$, thought they may or they may not, in any arbitrary commutative D.R.l. semigroup.

Lemma 16. $(a-b) \cup 0+(b-a) \cup 0=(a-b) \cup(b-a)$.

ProOF.

$$
0=a \cup b-a \cup b=\{0 \cup(b-a)\} \cap\{0 \cup(a-b)\}
$$

by (1.2) and (1.1). Since + is commutative,

$$
(a-b) \cup 0+(b-a) \cup 0=\{(a-b) \cup 0\} \cup\{(b-a) \cup 0\}=(a-b) \cup(b-a) .
$$

' $b$ ' is said to be metrically between $a$ and $c$ - in symbols, $(a, b, c) M-$ if and only if $a * b+b * c=a * c$. Since $L$ is distributive, there is already the notion of lattice betweenness - in symbols $(a, b, c) L-$ iff $a \cup c \geqq b \geqq a \cap c$. However in a Boolean-l-algebra these two notions are equivalent; in fact

THEOREM 3. In any commutative D.R.l.semigroup $A$ in which $a-(b \cup c)=(a-b) \cap(a-c)$, the following are equivalent.

1. $A$ is a Boolean-l-algebra

2. $(a, b, c) L$ if and only if $(a, b, c) M$

3. Metric betweenness has transitivity ' $t_{1}$ '

4. $(a, b, c) M$ and $(a, c, b) M$ imply $b=c$.

We need some lemmas in the proof of this theorem.

Lemma 17. For any $a, b, c$ in $L, a * b+b * c=a \cup c * b+b * a \cap c$.

PRoOf. $a \cup c * b+b * a \cap c=\{(a * b) \cup(c-b)\} \cap\{(b * c) \cup(a-b)\}+$ $\{(a * b) \cup(b-c)\} \cap\{(b * c) \cup(b-a)\}$ (by routine computation) $=$ $[\{(a * b) \cup(c-b)\} \cap\{(b * c) \cup(a-b)\}] \cup[\{(a * b) \cup(b-c)\} \cap$ $\{(b * c) \cup(b-a)\}]+[\{(a * b) \cup(c-b)\} \cap\{(b * c) \cup(a-b)\}] \cap$ $[\{(a * b) \cup(b-c)\} \cap\{(b * c) \cup(b-a)\}]=(a * b) \cup(b * c)+$ $(a * b) \cap(b * c)$ (again by routine computation) $=a * b+b * c$.

COROllary 8. $(a, b, c) M$ if and only if $(a \cup c, b, a \cap c) M$.

LEMMA 18. Under the hypothesis of theorem $3,(a, b, c) L$ implies $(a, b, c) M$.

PRoOF. Let $a a \cup c \geqq b \geqq a \cap c$. Then by lemma 15 [2], 


$$
\begin{aligned}
a * c=a \cup c-a \cap c & =(a \cup c-b)+(b-a \cap c) \\
& =a \cup c * b+b^{*} \mathrm{a} \cap c=a * b+b * c
\end{aligned}
$$

by corollary 8 . Hence $(a, b, c) M$.

Proof of THE Theorem 3. We shall prove that $1 \Rightarrow 2 \Rightarrow 3 \Rightarrow 2 \Rightarrow 1$ and $2 \Rightarrow 4 \Rightarrow 1$.

$1 \Rightarrow 2$. Let $(a, b, c) M$ then by corollary 8 , we can assume $a \geqq c$. Hence

$$
\begin{aligned}
a=(a-c)+c & =a * c+c=a * b+b * c+c \\
& \geqq(b-a)+(b-c)+c \geqq(b-a)+b .
\end{aligned}
$$

Hence by (1.2)

$$
0-(b-a)=b-\{b+(b-a)\} \geqq b-a \text { or } a \geqq b .
$$

So that by (1.4) $b \geqq c$ since $a-c \geqq a-b$, or $(a, b, c) L$; the other part follows from lemma 18.

$2 \Rightarrow 3$. Obvious.

$3 \Rightarrow 2$. See theorem 10.1 [9] and theorem 2.8 [5].

$2 \Rightarrow 1$. We have only to show that (1.4) holds in $A$ on account of lemmas $4,5,6$ and 8 of [2]. Let $a \geqq b \cup c$ and $a-b \geqq a-c$. Then

$$
\begin{aligned}
a * b=a-b & =a-(b \cap c)=a * b \cap c=a * b \cup c+b \cup c * b \cap c \\
& =a-(b \cup c)+b * c=a-c+c * b=a * c+c * b .
\end{aligned}
$$

Hence $(a, c, b) L$ or $c \geqq b$.

$2 \Rightarrow 4$. Since $2 \Rightarrow 1$, we shall show $1 \Rightarrow 4$. Let $(a, b, c) M$ and $(a, c, b) M$. Then $a \cup c \geqq b \geqq a \cap c$ and $a \cup b \geqq c \geqq a \cap b$. Hence $a \cup b=a \cup c$ and $a \cap b=a \cap c$ so that $b=c$.

$4 \Rightarrow 1$. Let $a \geqq b \cup c$ and $a-b \geqq a-c$. Since $(a, b, b \cap c) L$ we have

$a * b \cap c+b \cap c * b=a-(b \cap c)+b-b \cap c$

$$
\begin{aligned}
& =(a-b)+b-b \cap c=a * b+b * b \cap c=a * b \cap c \\
& =a-(b \cap c)=a-b=a * b,
\end{aligned}
$$

so that $(a, b \cap c, b) M$. Hence $b \cap c=b$ or $c \geqq b$. Therefore (1.4) holds in $A$ and consequently $\boldsymbol{A}$ is a Boolean-l-algebra.

An interesting application of the above theorem is the following.

COROLlaRY 9. If B is a Brouwerian algebra, with $a-(b \cup c)=(a-b) \cap(a-c)$ then the following are equivalent.

1) $B$ is a Boolean ring.

2) $(a, b, c) L$ iff $(a, b, c) M$.

3) $(a, b, c) M$ and $(a, c, b) M$ imply $b=c$.

4) Metric betweenness has transitivity $t_{1}$. 
From now on we simply write $(a, b, c)$ for $(a, b, c) L$ or $(a, b, c) M$ in $L$. The betweennes relation $(a, b, c)$ has the transitivities $t_{1}, t_{2}, \tau_{1}, \tau_{2}, T_{4}, T_{5}, T_{6}, T_{7}$ and $T_{1}, T_{2}, T_{3}, t_{3}$ iff $L$ is simply ordered (lemma 8.2, Th. 9.8 [9]).

A subset $S$ of $L$ is said to be a subgeometry iff $a, b \in S$ implies $a * b \in S$.

We shall treat any three distinct points $a, b, c \in L$ as the vertices of a triangle with sides $a * b, b * c$ and $c * a$ which will be denoted by $\Delta(a, b, c)$. A triangle is said to have fixty iff each vertex is equal to its opposite side. The following theorem throws some light on a $\Delta(a, b, c)$ to have fixty, in $L$.

THEOREM 4. The following are equivalent in $L$.

1) $\Delta(a, b, c)$ has fixty.

2) $a \cup b=b \cup c=a \cup c$ and $a \cap b \cap c=0$.

3) $\Delta(a * b, b * c, c * a)$ has fixty and $(0, a, b, c)$ is a subgeometry.

The proof of this theorem is contained in the next few lemmas.

Lemma 19. In any commutative D.R.l. semigroup $A$ in which $(a-b) \cap(b-a) \leqq 0,($ for all $a, b \in A)$ the following are equivalent.

1) $\Delta(a, b, c)$ has fixty.

2) $\Delta(a * b, b * c, c * a)$ has fixty and $(0, a, b, c)$ is a subgeometry.

Proof. That $1 \Rightarrow 2$ is obvious.

$2 \Rightarrow 1$ is routine (see Th. $2.16[5]$ ).

LEMMA 20. If $A$ is a commutative D.R.l. semigroup, such that $(a-b) \cap(b-a) \leqq 0$ for all $a$ and $b \in A$ then $\Delta(a, b, c)$ will have fixty if $a \cup b=b \cup c=a \cup c$ and $a \cap b \cap c=0$.

ProOf. Let $a \cup b=b \cup c=a \cup c$ and $a \cap b \cap c=0$. Then

$$
c+a \cap b=c \cup(a \cap b)=a \cup b
$$

and hence $a \cap b \geqq a \cup b-c=x$ (say), so that $x \cap c=0$. Also $x \cap(a * b)=0$ and

$$
x \cup(a * b)=a \cup b=x+c=x \cup c
$$

and hence $a * b=c$. Similarly the rest.

LEMMA 21. In any commutative D.R.I. semigroup $A$ in which $(a-b) \cap(b-a) \leqq 0$ for all $a$ and $b \in A$ if $\Delta(a, b, c)$ has fixty, then $a \cup b=$ $b \cup c=a \cup c$ and $a \cup b-a \cap b \cap c=a \cup b$.

Proof. Suppose $a=b * c, b=a * c$, and $c=a * b$. Then

$$
a \cup b=a * b+a \cap b=c+a \cap b \geqq c .
$$

Similarly $b \cup c \geqq a$ and $a \cup c \geqq b$. Hence $a \cup b=b \cup c=a \cup c$. Also $a \cup b-a \cap b \cap c=a \cup b \cup c-a \cap b \cap c$

$$
=(a * b) \cup(b * c) \cup(a * c) \text { (by direct computation) }=a \cup b .
$$


COROLlaRY 10. If $A$ is a commutativeD.R.l. semigroup in which $(a-b) \cap$ $(b-a) \leqq 0$, such that $a-a \cap b \cap c=a$ implies $a \cap b \cap c=0$, then $\Delta(a, b, c)$ has fixty iff $a \cup b=b \cup c=a \cup c$ and $a \cap b \cap c=0$.

COROLlaRY 11. If $B$ is a Brouwerian algebra such that $a-a \cap b \cap c=a$ implies $a \cap b \cap c=0$ then $B$ is a Boolean ring.

Proof. If $0 \leqq x \leqq a$, then

$$
a-a \cap x \cap(a-x)=(a-x) \cup\{a-(a-x)\}=(a-x) \cup a=a .
$$

Hence $x \cap(a-x)=0$ and so $x \cup(a-x)=a$ and so $B$ is section complemented and hence a Boolean ring.

THEOREM 5. There exists no equilateral triangle in $L$.

Proof. Let $a * b=b * c=a * c$. Then

$$
\begin{aligned}
a \cup b=a * b+a \cap b & =(b * c) \cup(c * a)+a \cap b \\
& \geqq\{(c-b) \cup(c-a)\}+a \cap b=\{c-(a \cap b)\}+a \cap b \geqq c .
\end{aligned}
$$

Similarly $b \cup c \geqq a$ and $a \cup c \geqq b$. Hence $a \cup b=b \cup c=a \cup c$. Now by 1.4 we have $a \cap b=a \cap c=b \cap c$ and so $a=b=c$. Hence equilateral $\Delta s$ will not exist in $L$.

THEOREM 6. L is a chain iff it is free of $\Delta s$ with fixty.

Proof. If $L$ is not a chain then there exist $a, b \in L$, such that $a$ is incomparable with $b$. Then $(a-b) \cup 0,(b-a) \cup 0$, and $(a-b) \cup(b-a)$ are three distinct points such that $\Delta((a-b) \cup \mathrm{O},(b-a) \cup 0,(a-b) \cup(b-a))$ has fixty (routine computation) contradicting the hypothesis. Hence if $L$ is free of triangles with fixty, then $L$ is a chain.

Conversely if $L$ is a chain then by theorem 4 , no triangle can have fixty.

One can easily observe that in the proof of the first part of the above theorem, we have not made use of (1.4). And so we have the following,

COROLlary 12. If $A$ is a commutative D.R.l. semigroup such that $(a-b) \cap$ $(b-a) \leqq 0$ (for all $a$ and $b)$, is free of triangles with fixty, then $A$ is a chain.

Following Blumenthal, Swamy introduced the notion of metrically convex Boolean-l-group (A Boolean- $l$-group is the direct product of a Boolean algebra and a lattice ordered group). See [5]: A Boolean-l-group is said to be metrically convex if and only if given $a \neq b$, then there is an $x$ such that $a \neq x \neq b$, and $a * x+x * b=a * b$. We extend this definition to Boolean-l-algebra and the following is an algebraic characterisation.

THEOREM 7. L is metrically convex iff it is atom free.

Proof. Let $L$ be metrically convex and $c>0$. Then there is an $x$, such that 
$(c, x, 0) M$. Hence $(c, x, 0) L$ or $c>x>0$. Hence $c$ is not an atom. Therefore $L$ is atom free.

If $L$ is atom free and $a$ and $c$ are distinct, then there is an $x$ such that $0<x<a * c$ and so $a \cap c \leqq x+a \cap c$ and $x+a \cap c=y$ (say). If $a \cap c=y$ then

$$
a * c=a \cup c-(x+a \cap c)=a * c-x
$$

hence by $1.4 x=0$. Similarly if $a \cup c=y$ then

$$
a * c=y-a \cap c=(x+a \cap c)-a \cap c \leqq x .
$$

Hence $a \cup c \neq y \neq a \cap c$ and $y$ lies metrically between $c$ and $a$.

Blumenthal introduced the notions of $B$-linearity and $D$-linearity in a Boolean geometry. An $n$-tuple $(n \geqq 3)$ of distinct points is said to be $B$-linear iff there exists a labelling $\left(p_{1} \cdots p_{n}\right)$ of its elements such that $B\left(p_{i}, p_{j}, p_{k}\right)$ hold where $1 \leqq i<j<k \leqq n$ and is said to be $D$-linear iff there exists a labelling $\left(p_{1} \cdots p_{n}\right)$ of its elements such that $d\left(p_{1} p_{n}\right)=\sum_{i=1}^{n-1} d\left(p_{i} p_{i+1}\right)$ where $d\left(p_{i}, p_{j}\right)$ is the metric operation and $B\left(p_{i}, p_{j}, p_{k}\right)$ means $p_{j}$ lies between $p_{i}$ and $p_{k}$ (lattice betweenness). There is no difficulty in extending this definition to Boolean-l-algebras and we state the following.

THEOREM 8. B-linearity implies D-linearity in $L$.

Proof. Routine, see 2.27 [5].

This theorem holds even in a commutative D.R.l. semigroup with $(a-b) \cap(b-a) \leqq 0$ for all $a$ and $b \in A$.

I take this opportunity to thank Prof. Dr. N. V. Subrahmanyam, for his valuable help in the preparation of this paper. I am indebted to the Referee, whose suggestions contributed much to the brevity of the paper.

\section{References}

[1] G. Birkhoff, Lattice Theory (Amer. Math. Soc. Col. Pub XXV, 1948).

[2] K. L. N. Swamy, 'Dually Residuated Lattice Ordered Semigroups', Math. Ann. 159, 105-114.

[3] K. L. N. Swamy, 'Dually Residuated Lattice Ordered Semigroups Il', Math. Ann. 160, $64-74$.

[4] K. L. N. Swamy, 'Dually Residuated Lattice Ordered Semigroup IIl', Math. Ann. 167, $71-74$.

[5] K. L. N. Swamy, Dually Residuated Lattice Ordered Semigroups (Doctoral Thesis, Andhra University, 196-).

[6] O. Wyler, 'Clans', Comp. Math. 17, 172-189.

[7] T. Nakano, 'Rings and Partly Ordered Systems', Math. Z. 99. 355-376.

[8] V. V. Rama Rao, 'On a Common Abstraction of Booleanrings and Lattice Ordered groups 1', Monat. Für. Math. 73, 411-421 (1969).

[9] Everett Pitcher and M. F. Smiley, 'Transitivities of Betweenness', T. Amer. Math. Soc. 52. (1942), 95-114.

Department of Mathematics (Arts College)

Andhra University Waltair (India) 